\title{
The Appearance of Tau Neutrinos from a Gamma Ray Burst
}

\author{
Nayantara Gupta 円 \\ Department of Theoretical Physics, \\ Indian Association for the Cultivation of Science, \\ Jadavpur, Kolkata 700 032, INDIA.
}

\begin{abstract}
The muon neutrinos from the Gamma Ray Bursts (GRBs) during their propagations oscillate to tau neutrinos. The tau neutrinos can be identified by the appearance of tau leptons which decay to muons below the detector. We examine the prospect of detecting these tau neutrinos from individual GRBs in a large area muon detector. We investigate for what range of physical parameters of the GRBs such as Lorentz factor, redshift (distance from the observer) and total energy released in neutrino emissions the appearance of tau neutrinos from individual GRBs will be observable in a ground based muon detector of $\mathrm{km}^{2}$ area. We find it is possible to detect unmistakeably the signature of tau neutrinos from individual GRBs in a ground based muon detector of $\mathrm{km}^{2}$ area above a muon threshold energy $30 \mathrm{PeV}$ for energies emitted by the GRBs in neutrinos $10^{53} \mathrm{erg}$ and $10^{54} \mathrm{erg}$ when their redshifts are below 0.01 and 0.03 respectively and they are observed at zenith angle $\theta=180^{\circ}$.
\end{abstract}

PACS number(s): $95.55 . \mathrm{Vj}$

\footnotetext{
${ }^{1}$ tpng@mahendra.iacs.res.in
} 


\section{Introduction}

In proton acceleration models the intrinsically produced tau neutrino flux is expected to be very small, typically a factor between $10^{-5}$ and $10^{-6}$ relative to electron and muon neutrino fluxes. The recent experimental measurements of atmospheric neutrinos suggest that neutrinos could just have vacuum flavor oscillations and the tau neutrino flux would be enhanced. In atmospheric neutrinos the contribution of tau neutrinos would be small but it would not be so incase of extragalactic neutrinos. The appearance of high energy tau neutrinos due to $\nu_{\mu} \rightarrow \nu_{\tau}$ oscillations can be observed by measuring the neutrino induced upward hadronic and electromagnetic showers or upward going muons.

The muon and electron neutrinos during their propagations through the Earth are absorbed due to charged current and neutral current interactions. We can expect an exponential fall of the number of muon or electron neutrinos at high energies due to absorptions after their passages through the Earth. The high energy tau neutrinos are also absorbed in the matter of the Earth but they are regenerated. The tau leptons produced from $\nu_{\tau} \mathrm{s}$ in charged current interactions again decay to produce $\nu_{\tau} \mathrm{S}$ of lower energies. The $\tau$ leptons produced from $\nu_{\tau} \mathrm{s}$ in charged current interactions decay in several channels. There are six decay modes of $\tau$ leptons described in [1].In all these decay modes $\nu_{\tau} \mathrm{s}$ are produced but in only one of these decay modes muons are produced which are expected to be detected by large area muon detectors.

It is possible to detect high energy tau neutrinos by their characteristic double shower events [2] or by the pileup of tau neutrino events near $100 \mathrm{TeV}$ [3]. It has been pointed out in [3] the observation of double-bang events is difficult in a first generation telescope such as AMANDA [4]. High energy tau neutrinos initiate a cascade in the Earth which will generate tau neutrinos of reduced energies in each interaction. Once the energies of the tau neutrinos fall below the threshold for absorption they propagate directly to the detector with energies in the range of $10 \mathrm{TeV}$ to $100 \mathrm{TeV}$. All $\nu_{\tau} \mathrm{s}$ with energies greater than $100 \mathrm{TeV}$ will have their energies reduced to about $100 \mathrm{TeV}$ after their passages through the Earth if the source neutrino energy spectrum falls slower than $E^{-2}$. In this way a pileup of tau neutrino events is expected to be produced near neutrino energy $100 \mathrm{TeV}$.

The very high energy $\nu_{\mu}$ neutrinos are absorbed as they penetrate the Earth. The $\nu_{\mu}$ neutrinos which pass through a lesser amount of matter inside the Earth are absorbed in lesser amount compared to the neutrinos which directly cross the diameter of the Earth. The muon neutrino events are maximum in the horizontal direction and minimum in the vertical direction in the muon detector. These events gradually increase from the vertical direction to the horizontal direction. But we expect the 
tau neutrino events to be uniformly distributed over all zenith angles in the muon detector because they are regenerated during their propagations through the Earth [3]. This is another experimental signature for $\nu_{\tau}$ neutrinos.

In this work we calculate the upward going muon event rates from muon and tau neutrinos of a GRB for very high muon threshold energies of the detector when the muon neutrinos are mostly absorbed during their propagations through the Earth. We can not confirm the observation of appearance of tau neutrinos in the muon detector unless we reach the threshold energy of the detector when the Earth is no more transparent to muon neutrinos. Also for neutrinos which are coming from horizontal directions the muon neutrinos are not significantly absorbed in the Earth. In this case the observation of muons even at very high energies does not provide conclusive evidence of the appearance of tau neutrinos from a single GRB. So the zenith angle at which the GRB is observed also plays a crucial role in providing unmistakeable signatures of the tau neutrinos in the muon detector from a single GRB. The proposed kilometer scale IceCube neutrino telescope from its South Pole location will observe a particular GRB at a fixed zenith angle. In that case we do not have the opportunity of observing uniform tau neutrino event rates at all zenith angles. We show that if we receive upward going muons at a very high threshold energy above $30 \mathrm{PeV}$ from a GRB then only we can confirm the appearance of tau neutrinos from that GRB. At such high threshold energies the role of atmospheric noise is negligible. So if we can separate the $\nu_{\tau}$ signals from $\nu_{\mu}$ signals of a GRB using the phenomena of $\nu_{\mu}$ absorptions during their propagations through the Earth we will be able to conclude about the appearance of tau neutrinos from that GRB. Also if the burst is bright and nearby then only we expect to detect muon signals from that burst.

We have not considered the tau neutrinos which produce double bang events in this work because they are much less compared to the events in which muons are produced via tau lepton decays $\left(\nu_{\tau} \rightarrow \tau \rightarrow \mu\right)[5]$.

A new effect has been pointed out in [6] that the $\nu_{\tau} \rightarrow \tau \rightarrow \nu_{\tau}$ regeneration process creates a secondary $\bar{\nu}_{\mu}$ flux. Though the $\bar{\nu}_{\mu}$ flux is at most 0.2 of the $\nu_{\tau}$ flux, the neutral current channels in total $\left(\bar{\nu}_{\mu}\right.$ and $\bar{\nu}_{e}$ channels) increase the detectability of $\nu_{\tau}$ events by $40 \%$.

We proceed as follows in this letter. In section 2 we mention about the procedure we have used for calculating the number of tau neutrinos from a GRB. The formulation used in this work to calculate the secondary muon events from tau neutrinos of a GRB has been discussed in section 3. The results of this work have been explained in section 4. We find it is possible to detect unmistakeably the signatures of tau neutrinos from individual GRBs in a ground based muon detector of $\mathrm{km}^{2}$ area above 
a muon threshold energy of $30 \mathrm{PeV}$ if the energy emitted by the GRB in neutrino emissions $\left(E_{G R B}\right)$ is of the order of $10^{53} \mathrm{erg}$ when its redshift is $z<0.01$ and $10^{54} \mathrm{erg}$ when its redshift is $z<0.03$ provided the GRB is at a zenith angle of $\theta=180^{\circ}$.

\section{The Number of Tau Neutrinos Produced from Muon Neutrinos of a Gamma Ray Burst}

We consider the fireball model of GRBs (Gamma Ray Bursts) [7] and calculate the expected tau neutrino event rates in a large area muon detector for different choices of the GRB parameters. Each GRB is characterised by its Lorentz boost factor $\Gamma$, total energy emitted in neutrinos $E_{G R B}$, distance of the GRB from the observer (red shift) $z$ and duration of the burst. Also there are other parameters like photon spectral break energy $E_{\gamma, \mathrm{MeV}}^{b}$ and wind variability time $t_{v}$. There is correlation between the parameters minimum Lorentz factor $\Gamma$, wind variability time $t_{v}$ and $E_{\gamma, \mathrm{MeV}}^{b}$ for known values of wind luminosity and equipartition parameter $\epsilon_{B}$ [8]. We have used this correlation in calculating the number of neutrinos emitted from a GRB. The detail procedure for calculation of number of muon neutrinos produced from a GRB has been discussed in [9]. The original muon neutrinos from GRBs are undergoing oscillations during propagation and tau neutrinos are produced in this way. The probablity of oscillation from $\nu_{\mu}$ to $\nu_{\tau}$ is given by

$$
\operatorname{Prob}\left(\nu_{\mu} \rightarrow \nu_{\tau}\right)=\sin ^{2} 2 \theta \sin ^{2}\left(\frac{\triangle m^{2} d}{4 E_{\nu}}\right)
$$

Considering the values of neutrino mass difference and mixing as found from SuperKamiokande data $\left(\sin ^{2} 2 \theta \sim 1, \triangle m^{2} \sim 10^{-3} \mathrm{eV}^{2}\right)$ [10] and the distance $(d)$ of the source of the order of a megaparsec to thousands of megaparsecs the above expression for probablity averages to about half for all relevant neutrino energies to be considered for detection [1]. The number of high-energy muon neutrinos is suppressed by a costant factor of half independent of its energy due to neutrino oscillations. The number of muon neutrinos produced from a GRB when multiplied by the probablity of oscillation from muon neutrinos to tau neutrinos gives the number of tau neutrinos produced from that GRB.

Number of $\nu_{\tau}$ s produced from a GRB due to $\nu_{\mu} \rightarrow \nu_{\tau}$ oscillation $=$ Number of $\nu_{\mu} \mathrm{s}$ from the GRB $\times \operatorname{Prob}\left(\nu_{\mu} \rightarrow \nu_{\tau}\right)$ 


\section{The Number of Secondary Muons Produced from Tau Neutrinos and Muon Neutrinos of a GRB}

The tau neutrinos produce tau leptons in charged current interactions during their passages through the Earth. The energy dependent charged current cross-sections for productions of tau leptons from tau neutrinos and muons from muon neutrinos have been used from [11]. The energy dependent total cross-sections for muon neutrinos absorptions have also been used from [11]. We have calculated the number of secondary muons from a single GRB produced via the decays of the tau leptons from $\nu_{\tau} \mathrm{s}$ using the formula for muon event rate calculation discussed in [1]. The number of muon events has been calculated as a function of the muon threshold energy of the detector of $\mathrm{km}^{2}$ area. The probablity of the decay process $\tau \rightarrow \nu_{\tau} \mu \bar{\nu}_{\mu}$ in which a muon will be produced is 0.18 [目]. The tau lepton carry a fraction 0.75 of the initial tau neutrino energy and the muon produced from $\tau$ decay carry a fraction 0.4 of the tau lepton energy [12, 1, 6]. The secondary muons produced from $\tau$ decays below the detector lose energy during their propagation. We have used the formula for energy loss of muons in rock from [13] in calculating the energy dependent ranges of the muons in rock. The procedure for calculation number of secondary muons produced from muon neutrinos of a GRB has been discussed in 99.

\section{Results and Discussions}

We do a study on the appearance of tau neutrinos from a single GRB for the first time. Each gamma ray burst is an individual phenomenon of short duration in the Universe. The observation of the tau leptons from GRBs can probe studies on neu-

trino oscillations with $\triangle m^{2}$ to a value as low as $10^{-17} \mathrm{eV}^{2}$ [3]. Here the square of the mass difference is relative to the original $\nu_{e, \mu}$. This kind of an observation on $\nu_{\tau}$ appearance would extend the present understanding of neutrino oscillations $\left(\nu_{\mu} \rightarrow \nu_{\tau}\right)$ by fourteen orders of magnitude. Neutrino astronomy with tau neutrinos also opens an opportunity to know about the ultra high energy neutrino productions from different astrophysical objects. As has been discussed in [3] if there exist large angle vacuum neutrino oscillations involving $\nu_{\tau}$ with sufficient value of $\triangle m^{2}$ consistent with SuperKamiokande results [15] a pure $\nu_{\mu}$ source of energy upto even $10^{21} \mathrm{eV}$ can be observed in the muon detector. Since these neutrinos are of very high energy the final $\nu_{\tau} \mathrm{s}$ always point back to their source.

In Fig.1. the number of muon events from muon neutrinos and tau neutrinos of a GRB have been plotted for different muon threshold energies of the muon detector 
of $k m^{2}$ area. The energy of the GRB is assumed to be $10^{54} \mathrm{erg}$ and the burst is observed at zenith angle $\theta=180^{\circ}$. The GRB is at a distance of redshift $z=0.03$. The wind luminosity is assumed to be $10^{53} \mathrm{erg} / \mathrm{sec}$. Nearly $10 \%$ of the initial fireball proton energy or wind energy is released in neutrino emissions from a GRB [9]. The Lorentz factor of the GRB is assumed to be 199.52 for photon spectral break energy $E_{\gamma}^{b}=50.118 \mathrm{MeV}$ [8]. We are interested to know for what muon threshold energy of the detector the number of muon events from tau neutrinos of a GRB outnumber the number of muon events from muon neutrinos of that GRB. We have varied the threshold energy of the muon detector upto $100 \mathrm{PeV}$. As we increase the muon threshold energy above $20 \mathrm{PeV}$ we observe the number of muon events from tau neutrinos remain constant at a value of nearly levent $/ \mathrm{km}^{2}$. Near muon threshold energy $30 \mathrm{PeV}$ the number of muon events from tau neutrinos of a GRB is more than the number of muon events from muon neutrinos of that GRB. Also the signal from tau neutrinos does not drop below 1event $/ \mathrm{km}^{2}$. In Fig.1. we have also plotted the sum of muon neutrino and tau neutrino events from the GRB in a muon detector of $\mathrm{km}^{2}$ area against the muon threshold energy of the detector.

In Fig.2. the number of muon events from muon neutrinos and tau neutrinos of a GRB in a muon detector of $\mathrm{km}^{2}$ area have been again plotted as a function of muon threshold energy of the detector. The GRB is assumed to be at a distance of redshift $z=0.01$ and total energy emitted in neutrinos is $E_{G R B}=10^{53} \mathrm{erg}$. The mean isotropic equivalent energy emitted by GRBs is of the order of $10^{53} \mathrm{erg}$, this result has been obtained in [16]. The allowed values of Lorentz factors and corresponding photon spectral break energies have been used from [B] . An initial tau neutrino threshold energy of $100 \mathrm{TeV}$ corresponds to a secondary muon threshold energy of $30 \mathrm{TeV}$ because the muons produced via decays of tau leptons produced from tau neutrinos carry about $3 / 10$ of the initial tau neutrino energy [6]. In this plot we find that near muon threshold energy $30 \mathrm{PeV}$ the number of muon events from tau neutrinos of a GRB remains near 1event $/ \mathrm{km}^{2}$ but the number muon events from muon neutrinos of that GRB falls below that. When the order of the muon threshold energy is $\mathrm{PeV}$ the number muon events from individual GRBs are not sensitive to the value of minimum Lorentz factor.

In Fig.3. the dependence of muon events from tau neutrinos and muon neutrinos of a GRB on the distance of the GRB from the observer has been shown. The energy emitted in a burst is assumed to be $E_{G R B}=10^{54} \mathrm{erg}$. The number of muon events falls rapidly as we increase the redshift of the GRB from the observer. The detector threshold energy is assumed to be $E_{t h}=30 \mathrm{PeV}$ and the zenith angle of observation is $\theta=180^{\circ}$. The GRBs are distributed uniformly upto a redshift of about $z=2$ [17]. There is only opportunity of detecting tau neutrino appearance from bursts at very 
low redshifts. From Fig.3. we notice that at all redshifts the number muon events from muon neutrinos and tau neutrinos of a GRB remain almost equal for muon threshold energy $E_{t h}=30 \mathrm{PeV}$.

The number of muon events both from tau neutrinos and muon neutrinos of a GRB increase linearly with increasing values of $E_{G R B}$, the total energy emitted in neutrino emissions.

We also find from our calculations that when $E_{G R B}=10^{53} \mathrm{erg}$ and $E_{G R B}=10^{54} \mathrm{erg}$ we can not expect to detect tau neutrino signals from individual GRBs in $\mathrm{km}^{2}$ area muon detector if their distances are more than $z=0.01$ and $z=0.03$ respectively. We have used the allowed values of minimum Lorentz factor and photon spectral break energies for wind luminosity $10^{53} \mathrm{erg} / \mathrm{sec}$ from [8]. The wind durations are typically of the order of 10 seconds. One can find similar constraints for detectability of tau neutrinos from individual GRBs in $\mathrm{km}^{2}$ area muon detector for other values of $E_{G R B}$ which is the total energy emitted in neutrino emissions using the allowed values of GRB parameters minimum Lorentz factors, photon spectral break energies and wind luminosities corresponding to those values of $E_{G R B}$.

\section{Conclusion}

The detectability of tau neutrinos produced from oscillations of muon neutrinos originated from individual GRBs has been tested for the first time in a muon detector of $\mathrm{km}^{2}$ area. The role of the GRB parameters on the detectability of tau neutrinos from individual GRBs has been explained in this work. If the energies emitted in neutrino emissions is $10^{53} \mathrm{erg}$ and $10^{54} \mathrm{erg}$ and the bursts are at less than distances of redshift $z=0.01$ and $z=0.03$ respectively it will be possible to detect $\nu_{\tau}$ signals from them in $\mathrm{km}^{2}$ area muon detector above muon threshold energy $30 \mathrm{PeV}$ when the bursts are at a zenith angle of $\theta=180^{\circ}$. Also the oscillation parameters influence the number of $\nu_{\tau}$ events. One can find out the values of neutrino mass difference and mixing parameters in extragalactic neutrinos by observing the disappearance of muon neutrinos or the appearance of tau neutrinos.

\section{Acknowledgment}

The author is thankful to the referee for helpful suggestions. 


\section{References}

[1] S. I. Dutta, M. H. Reno and I. Sarcevic, Phys. Rev. D 62, 123001 (2000). S. Iyer, M. H. Reno and I. Sarcevic, Phys. Rev. D 61, 053003 (2000).

[2] H. Athar, G. Parente and E. Zas, Phys. Rev. D 62, 093010 (1998).

[3] F. Halzen and D. Saltzberg, Phys. Rev. Lett. 81, 4305 (1998).

[4] See, for example, URL http://amanda.berkeley.edu/

[5] J. Alvarez-Muñiz, F. Halzen and D. W. Hooper, Phys. Rev. D 62, 093015 (2000).

[6] J. F. Beacom, P. Crotty and E. W. Kolb, astro-ph/0111482

[7] E. Waxman and J. Bahcall, Phys. Rev. Lett. 78, 2292 (1997).

[8] D. Guetta, M. Spada and E. Waxman, Astrophys. J. 559, 101 (2001).

[9] N. Gupta, astro-ph/0201509, Phys. Rev. D in press.

[10] SuperKamiokande Collaboration, Y. Fukuda et al. Phys. Rev. Lett. 81, 1562 (1998).

[11] R. Gandhi, C. Quigg, M. H. Reno and I. Sarcevic, Phys. Rev. D 58, 093009 (1998).

[12] T. K. Gaisser, CosmicRaysandParticlePhysics, Cambridge University Press (1992).

[13] A. Dar, J. J. Lord and J. Wilkes, Phys. Rev. D 33, 303 (1984).

[14] V. Agrawal, T. K. Gaisser, P. Lipari and T. Stanev, Phys. Rev. D 53, 1314 (1996).

[15] Y. Fukuda et al., Phys. Lett. B 433, 9 (1998).

[16] D. A. Frail et al., Astrophys. J. 562, L55 (2001)

[17] T. Piran, astro-ph/0111314. 


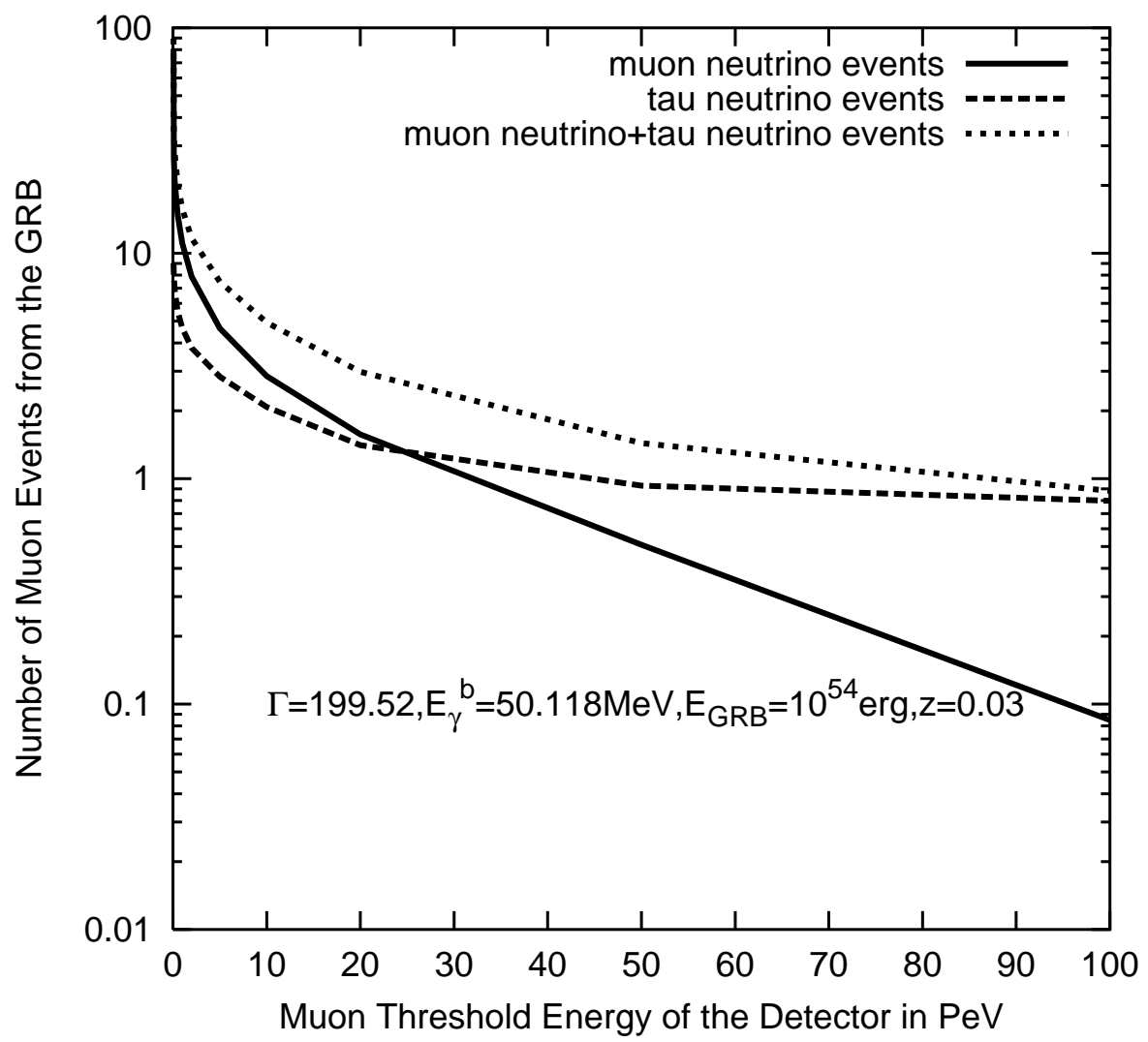

Figure 1: Number of secondary muon events produced from tau neutrinos of a GRB via tau lepton decays $\left(\nu_{\tau} \rightarrow \tau \rightarrow \mu\right)$ in a muon detector of $\mathrm{km}^{2}$ area and also the muon events from muon neutrinos of that GRB have been plotted in this figure for various threshold energies of the detector. The GRB is at a zenith angle of $\theta=180^{\circ}$. The wind luminosity of the GRB is assumed to be $10^{53} \mathrm{erg} / \mathrm{sec}$ for equipartition parameter $\epsilon_{B}=0.01$. The correlation of GRB parameters $\Gamma$ and photon spectral break energy $E_{\gamma, \mathrm{MeV}}^{b}$ have been used from [Q] $]$. The total energy emitted in each burst is assumed to be $E_{G R B}=10^{54} \mathrm{erg}$ and the redshift of the burst is $z=0.03$. The sum of muon neutrino and tau neutrino events in the muon detector have also been shown in this figure for different muon threshold energies. 


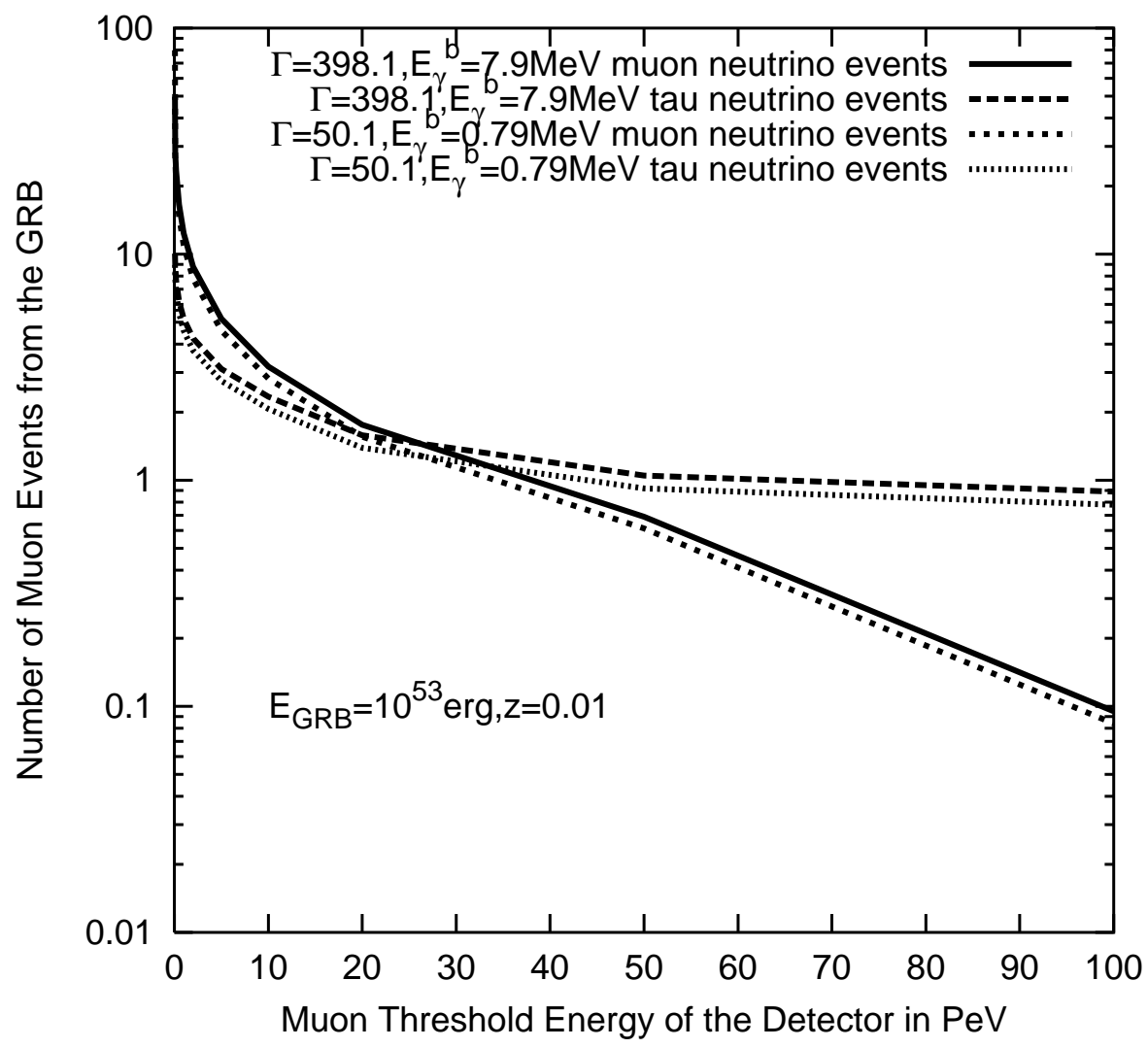

Figure 2: The number of muon events from $\nu_{\tau} \mathrm{s}$ and $\nu_{\mu} \mathrm{s}$ of a GRB in $\mathrm{km}^{2}$ area have been plotted as a function of muon threshold energy of the detector. We have not considered the double bang events in our calculations. The burst is at $\theta=180^{\circ}$ and wind luminosity of the GRB is assumed to be $10^{53} \mathrm{erg} / \mathrm{sec}$ for equipartition parameter $\epsilon_{B}=0.01$. The total energy emitted in neutrinos is assumed to be $E_{G R B}=10^{53} \mathrm{erg}$. Here $\Gamma$ is the Lorentz factor, and $E_{\gamma, M e V}^{b}$ is the observed photon spectral break energy. Their allowed values are taken from [8] 


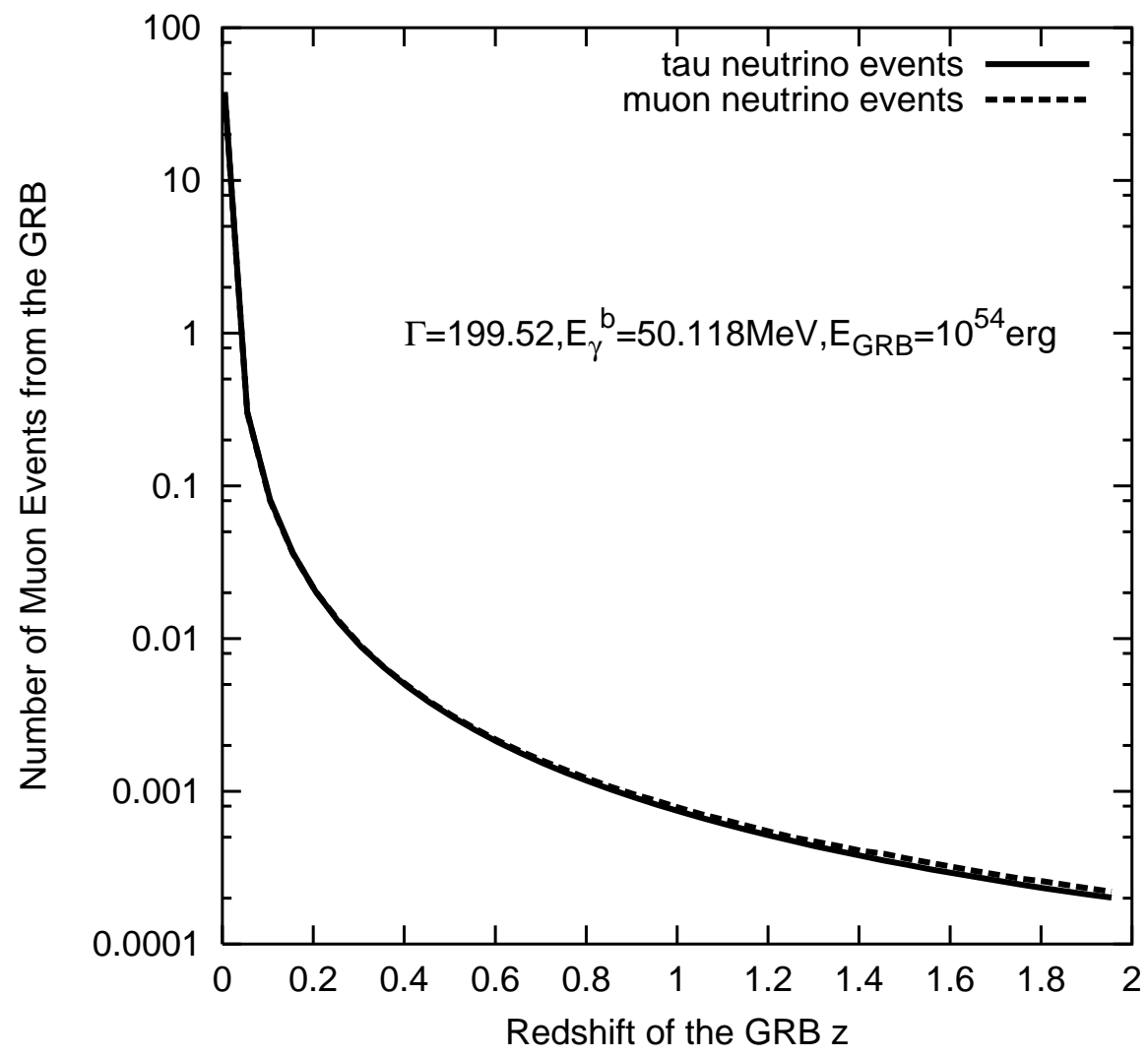

Figure 3: The dependence of number of muon events produced via tau lepton decays $\left(\nu_{\tau} \rightarrow \tau \rightarrow \mu\right)$ from tau neutrinos of a GRB and also from muon neutrinos of that GRB on the redshift of the GRB has been shown in this figure. The area of the muon detector is assumed to be $1 \mathrm{~km}^{2}$. The GRB is at zenith angle $\theta=180^{\circ}$. The wind luminosity of GRB is assumed to be $10^{53} \mathrm{erg} / \mathrm{sec}$ and equipartition parameter $\epsilon_{B}=0.01$. The threshold energy of the detector is assumed to be $E_{t h}=30 \mathrm{PeV}$. 